U.D.C. 330.42

keywords: regional economy, demand, supply, sustainability, chaotic states, strange attractor

L. A. Korshunov, G. P. Bystrai

\title{
DETERMINATE NONPERIODIC VARIATION OF THE GROSS REGIONAL PRODUCT
}

The classical problem of product supply and demand, which was first defined by A. Marshall, is reduced to Lorentz model in a nonlinear statement and it describes sustainable circular processes of commodity market, including price range increment in the context of crisis forthcoming (inflation); destabilization due to nonmanufacturing costs; the main stages of crisis development, including a stage of chaotic behavior of residence time at each attractor (equilibrium point); possibility of surmounting the crisis by means of modification of certain parameters. The given approach is used for the purpose of construction of a qualitative model of commodity price readjustment during precrisis and crisis periods, and finding reliable prognostication time.

A. Marshall established a mechanism of supply and demand ratio influence on commodity pricing in the context of perfect competition [6]. Supply and demand are interrelated with price: demand, as a rule, decreases under price advance and grows under its reduction; supply grows with price advance in most cases and decreases under price reduction. Therefore, stable price (equilibrium price) is set at the point, where demand is balanced with supply (i.e. at the cross point of supply and demand curves in graphic portrayal). Price that exceeds the equilibrium price, results in excess of supply over demand, which inevitably leads to price reduction. Price that appeared to be lower than the equilibrium price results in excess of demand over supply, which leads to price increase. Only with equilibrium price $X^{0}$ demand is balanced with supply (balanced in terms of the fact that incentives for modification of three functionally related values - demand, supply and price do not occur). Disturbance of the balance between supply and demand for goods and services

\footnotetext{
1 The research work was financed partly on account of the purpose-oriented Programme by the Ural Branch of the Russian Academy of Sciences; the programme is aimed at supporting the interdisciplinary projects implemented jointly with the scientists from the Siberian and the Far-Eastern Branches of the Russian Academy of Sciences within the framework of the integration project «Social and economic immunity of the region: diagnostics and forecast of crisis phenomena protectability» (project №09-C-6-1001 «Diagnostics of condition, trend simulation and forecast of regional development in Russia until 2030»).
}

might be one of the aspects referred to an economic crisis. Particularities of crisis condition generation within the formalized knowledge database of volatile market attract not only its participants - consumers and salespeople, but also practising economists, as well as orthodox economics and economic synergy representatives; a contrastive analysis related to the given aspects is given in $[1,6]$. The present research work is dedicated to analysis into the regional market of Gross Regional Product (GRP), although initially the task is set with regard to any commodity market in the context of conception by A. Marshall.

Unbalancedcommoditymarket. Nonmanufacturing costs. Formalized prediction model. Let us consider that supply and demand quantity under weak deflection from the equilibrium point (fig. 1) in the context of unrestrained competition at a certain point of time is described by equations

$$
\begin{gathered}
J_{e}=J^{0}+\delta J_{e}, \quad J_{i}=J^{0}+\delta J_{i}, \\
\delta J_{e} \equiv<\overline{\Delta J_{e}}>, \quad \delta J_{i} \equiv<\overline{\Delta J_{i}}>.
\end{gathered}
$$

Increments - the last summands in (1) - can have a different sign. The given increments referred to commodity quantities are average in terms of small time gap and average in terms of all consumers and producers respectively. Assuming supply and demand are interdependent, we come to a variation scheme of nonequilibrium process specification:

$$
\begin{gathered}
\delta J_{e}=L_{e e}^{0} \delta X_{e}+L_{e i}^{0} \delta X_{i}, \delta J_{i}=L_{i e}^{0} \delta X_{e}+L_{i i}^{0} \delta X_{i}, \\
X_{e}=X^{0}+\delta X_{e}, X_{i}=X^{0}+\delta X_{i},
\end{gathered}
$$

here $X^{0}-$ a conjectural equilibrium price (supply and demand prices are equal to each other). Coefficients $L_{e e}^{0}$ and $L_{i i}^{0}$ in (2) are termed as lines, a $L_{i e}^{0}, L_{e i}^{0}-$ transverse elasticity coefficients: $L_{i e}^{0}=\delta J_{i} / \delta X_{e}, \quad L_{e i}^{0}=\delta J_{e} / \delta X_{i}, \quad L_{e e}^{0}=\delta J_{e} / \delta X_{e}$, $L_{i i}^{0}=\delta J_{i} / \delta X_{i}$, calculated by way of variations, and which might be normalized with quantity and price value at the equilibrium point. Index «0» here and further will correspond to an equilibrium point.

Price increments are average for the considered local time gap as well, and average in terms of all consumers and producers respectively: $\delta X_{e} \equiv<\overline{\Delta X_{e}^{t}}>, \delta X_{i} \equiv<\overline{\Delta X_{i}^{t}}>$. Dimensions of the given values are as follows: $[X]=$ rub./c.c.u. (price of one commodity conventional unit $),[J]=$ c.c.u./month (commodity quantity per time unit). 


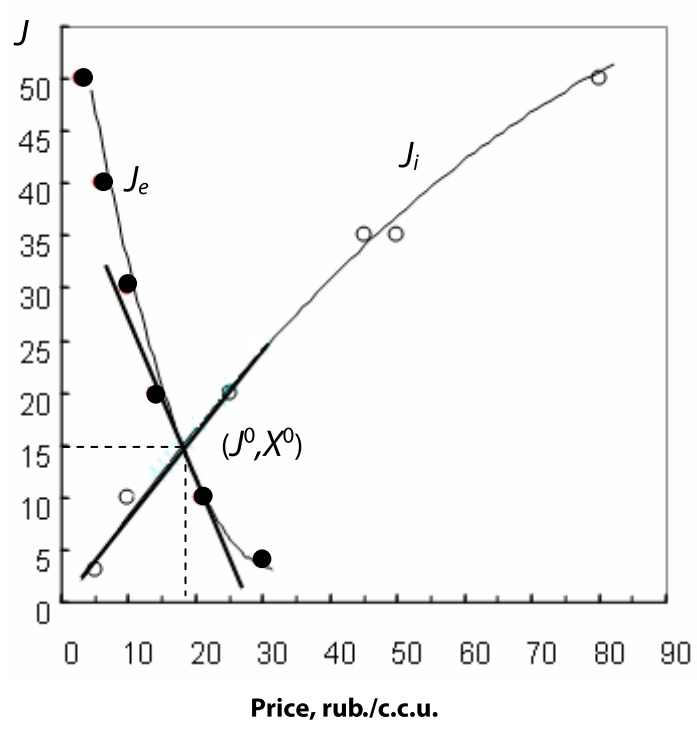

Fig. 1. Representative diagram for commodity supply and demand in the context of unrestrained competition; along with the points, the lines approximating them are also specified on the diagram. The curves intersect at the equilibrium point, where the price is equal to $X^{\circ}$, and quantity - $J^{\circ}$. In the neighbourhood of the given point supply and demand are price affine functions, and that is reproduced by lines

At equilibrium (at a fixed point) the quantity of demand is equal to the quantity of supply $J_{e}=J_{i}=J^{0}$. Combined linear equations (1-2) link supply and demand and respective prices in non-equilibrium tasks and correspond to the market economy classical scheme introduced by A. Marshall. In a linear task, where demand affects supply in the same manner as supply affects demand, cross coefficients are symmetric: $L_{i e}^{0}=-L_{e i}^{0}$. The presence of the minus mark is subject to different dependence of supply and demand on price (fig. 1). In nonlinear tasks the specified coefficients (or at least several of them) are dependent on prices [1].

In fig. 1 a representative diagram for commodity supply and demand is given, where the evaluated values are marked by points. Values of the price in equilibrium and commodity quantity are restored from the inverse task solution, for instance, for the given curves: $X^{0}=17,4$ rub./c.c.u., $J^{0}=14,5$ thousand c.c.u./day, with elasticity coefficients $L_{i i}^{0}=2, L_{i e}^{0}=1,2, L_{e i}^{0}=-1,2, L_{e e}^{0}=-3$.

Lorenz's strange attractor for a nonlinear market by Marshall. Let us discuss a set of three nonlinear differential equations for a classical supply and demand market at a specific commodity market in terms of the most simple problem definition, assuming it has at least one equilibrium point (fixed point) and well-known elasticity coefficients.

1. Let us consider that supply price velocity is generated due to a certain potential market field with function $\mathrm{G}: \dot{X}_{i}=-\varepsilon\left(\partial G / \partial X_{i}\right)$; it is dependent on demand, supply and corresponding prices, $\varepsilon-$ dimension constant. The right part of the equation can be represented as a gradient from the given function $G=-J_{e} X_{e}+J_{i} X_{i}+\sigma$ (profit with a reversed sign), which also includes nonmanufacturing costs $\sigma$. The difference between supply and demand with $\sigma$ available for the given time point can be represented as an expression $P=J_{e} X_{e}-J_{i} X_{i}-\sigma$. Let us take into consideration a certain coefficient $\chi \geq 1$, which determines the given costs as a certain share of production costs: $\sigma=(\chi-1) J_{i} X_{i}$; with $\chi=1$ the lower bound is $\sigma=0$. The upper bound is not specified, however, the cases with very large values $\mathrm{c}$ are unlikely to occur. A cost function in a linear task is quadratic function for price and it is determined [1] by a quadric quantic $G=-L_{e e}^{0} X_{e}^{2}-L_{e i}^{0} X_{e} X_{i}+\chi\left(L_{i e}^{0} X_{e} X_{i}+L_{i i}^{0} X_{i}^{2}\right) ; \quad$ its gradient is equal to $G_{X_{i}}=\left(-L_{e i}^{0}+\chi L_{i e}^{0}\right) X_{e}+2 \chi L_{i i}^{0} X_{i}$, then $\dot{X}_{i}=-2 \chi L_{i i}^{0} \varepsilon X_{i}+\left(L_{e i}^{o}-\chi L_{i e}^{0}\right) \varepsilon X_{e}$. If we take the variational derivative from the left and right parts of the given equation, we obtain the following variation equation for deflection rate of cost price from $X^{0}$ :

$$
\delta \dot{X}_{i}=-\alpha\left(\delta X_{i}\right)+\beta\left(\delta X_{e}\right),
$$

where $\alpha=2 \chi L_{i i}^{0} \varepsilon>0, \beta=\left(L_{e i}^{0}-\chi L_{i e}^{0}\right) \varepsilon$.

2. The second variation equation, which has a derivative in its left part, specifies time variation for demand price perturbance on the market:

$$
\delta \dot{X}_{e}=-\gamma\left(\delta X_{e}\right)+c L_{i i}\left(\delta X_{i}\right), \gamma=1 / \tau_{\delta X e},
$$

where $L_{i i}$ - supply elasticity coefficient based on supply price, $\gamma$ and $c$-certain parameters; $\tau_{\delta \text { Xe }}$ - demand price relaxation time relative to a new equilibrium point.

3. Let us consider a market condition, under which only the supply elasticity coefficient based on supply price $L_{i i}$ is variable out of all four elasticity coefficients within matrix $\left\|L_{i e}\right\|$. Therefore, let us enter the following variation equation for it:

$$
\begin{gathered}
\delta \dot{L}_{i i}=-\Gamma \delta L_{i i}+k\left(\delta X_{i}\right)\left(\delta X_{e}\right), L_{i i}=\delta J_{i} / \delta X_{i}, \\
\delta L_{i i} \equiv L_{i i}^{00}-L_{i i}, \Gamma=1 / \tau_{L},
\end{gathered}
$$

where $L_{i i}^{00}-$ a certain coefficient of the elasticity line, and deflection from it is considered by equation (5), $k$ - a certain coefficient, $\tau_{L}$ - relaxation time of elasticity coefficient under transition to a new equilibrium point.

Reduction of the equation system to Lorentzequation system. In order to simplify the form of equations, let us substitute variables $\delta X_{i}, \delta X_{e}, L_{i i}$ for new variables $x, y, w$, and parameters $\Gamma, \gamma, \alpha, \beta, k, c, L_{i i}^{0}-$ for parameters $b, l, r$. We consider the following ratios to be valid: $t \equiv \gamma t, x=\delta X_{i} / X^{0}, y=(\beta / \alpha)\left(\delta X_{e} / X^{0}\right)$, 


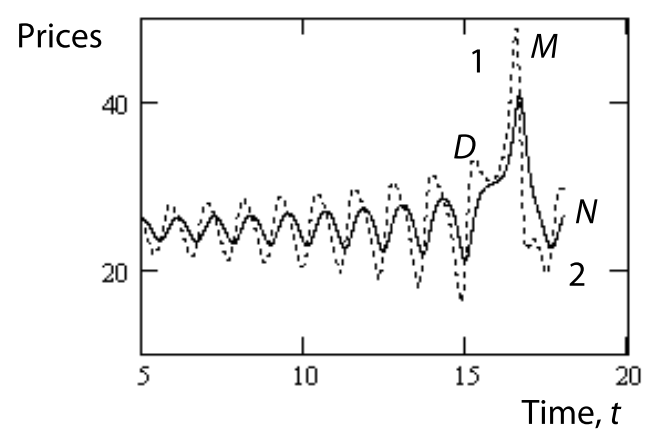

a

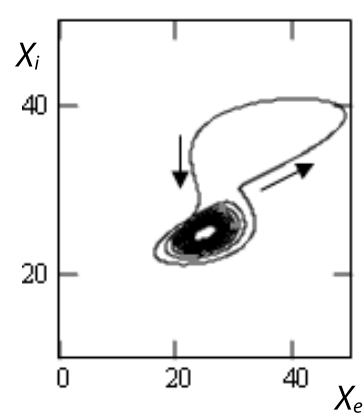

b

Fig. 2. Dynamics of transition to crisis state and crisis turnaround at the first time stage: 1 - demand prices (consumer prices, c.u.), 2 - supply prices (producer prices c.u.) with $I=2 \chi L_{i i}^{0} \varepsilon / \gamma=3, r=(\beta / \alpha)\left(c L_{i i}^{00} / \gamma\right)=29$.. D - crisis origin, $M$ - crisis peak, $N$-crisis turnaround

$\omega=(\beta / \alpha)\left(c L_{i i} / \gamma\right) ; \quad X^{0}=\gamma / \sqrt{k c}, \quad b=\Gamma / \gamma$, $l=2 \chi L_{i i}^{0} \varepsilon / \gamma, r=(\beta / \alpha)\left(c L_{i i}^{00} / \gamma\right)$. For the given supply and demand prices we have respectively:

$$
X_{e}^{*}=1+\alpha y / \beta, X_{i}^{*}=1+x,
$$

where $X_{i}^{*}=X_{i} / X^{0}, X_{e}^{*}=X_{e} / X^{0}$.

We enter supply and demand curves (1) as nonlinear dimensionless expressions:

$$
J_{e}^{*}=1+L_{e e}^{0^{*}} y+L_{e i}^{0^{*}} x, J_{i}^{*}=1+L_{i e}^{0^{*}} y+A(1+B z) x,(6)
$$

here

$J_{e}^{*}=\frac{J_{e}}{J^{0}}, L_{e e}^{0^{*}}=\frac{\alpha L_{e e}^{0} X^{0}}{\beta J^{0}}, L_{e i}^{0^{*}}=\frac{L_{e e}^{0} X^{0}}{J^{0}} ; L_{i e}^{0^{*}}=\frac{\alpha L_{i e}^{0} X^{0}}{\beta J^{0}}$, $A=\frac{L_{i i}^{00} X^{0}}{J^{0}}, B=\frac{\gamma}{c L_{i i}^{00}} ; J_{i}^{*}=\frac{J_{i}}{J^{0}}$,

$L_{i i}^{*}=\frac{\left(L_{i i}^{00}+\delta L_{i i}\right) X^{0}}{J^{0}}=A(1+B z) x, z=\frac{c}{\gamma} \delta L_{i i}$.

In the issue, equations (4)-(5) are reduced to a simple form $\dot{x}=l(y-x), \quad \dot{y}=-y+x \omega$, $\dot{\omega}=-b \omega+b r-x y$ and they can be reduced to Lorentz equations $[1,4]$ with $\omega=r-z$

$$
\dot{x}=l(y-x), \dot{y}=-y+r x-x z, \dot{z}=-b z+x y \text {; }
$$

The latter can be solved by numerical procedures.

In equation (7) the new variable is equal to fractional variation of elasticity coefficient: $z=r-\omega=(c / \gamma) \delta L_{i i} \equiv \delta L_{i i} / L_{i i}^{0}$. Value $\gamma / c$ has dimension of the elasticity coefficient. This implies condition of equilibrium for equation (3): $c L_{i i}^{0} / \gamma=1$, $B=1 / r$. With high values of $r-$ parameter $B \sim 1$. For instance, with $r=1$ parameter $B=1$; with $r \sim 30, B \sim 0,03$. Condition of equilibrium for (1) produces $\alpha / \beta=1$.

Thus, in terms of interpretation of the suggested nonlinear market scheme variable $x$ responds to deviation of the supply price from the equilibrium value, $y-$ to the corresponding deviation of demand price, and variable $z$ specifies fractional variation of supply elasticity coefficient based on supply price.
Numerical work results. Strange attractor of the market economy. At the outset, let us consider a hypothetical market with the lack of nonproduction $\operatorname{costs} \sigma=0, \chi=1$. In the issue of numerical calculations within Lorentz equation system (7), we obtain strange attractor [1] with parameter values $b=8 / 3, l=10$, $r=29$ within a wide domain of variation of entry conditions, when the main path, which is located at the existing point of equilibrium (more correctly, named fixed points [7]), leaves its domain of attraction and is directed towards another attractor (fig. 2). Attractor is a domain of attraction of phase paths.

During the following time stages the main path «rushes» between two attractors and jumps in a noncyclic nature. In the vicinity of each of the fixed points a hypothesis by A. Marshall referred to interdependence of demand, supply and corresponding prices with elasticity coefficients is valid. Transition to a new point of equilibrium (crisis) is of major interest.

Such response by the solutions obtained was given the name determinate chaos $[1,7,8]$, so far as chaos occurs not due to noise effect in the right parts of the obtained equation system, but owing to system nonlinear attributes. Crisis begins with a sudden demand price advance (domain $D$ in fig. 2a), subsequently cost prices grow, and the elasticity coefficient of supply scales back to its bottom limit and converges to the pre-crisis values. In domain $M$ the peak of the crisis is observed. A pre-crisis condition is specified by a circular annual process. Domain $N$ refers to crisis turnaround. Then (at the third time stage) cycles with small price amplitude follow; they are not assayed in the present paper. In fig. 2 theoretical time dependence regarding supply and demand prices upon time at the first time stage are given; in fig. 3 - industrial producer prices in the Altai Territory $\left(X_{i}\right)$ and consumer prices $\left(X_{\mathrm{e}}\right)$ for the given products in 1999-2008 [2]. 

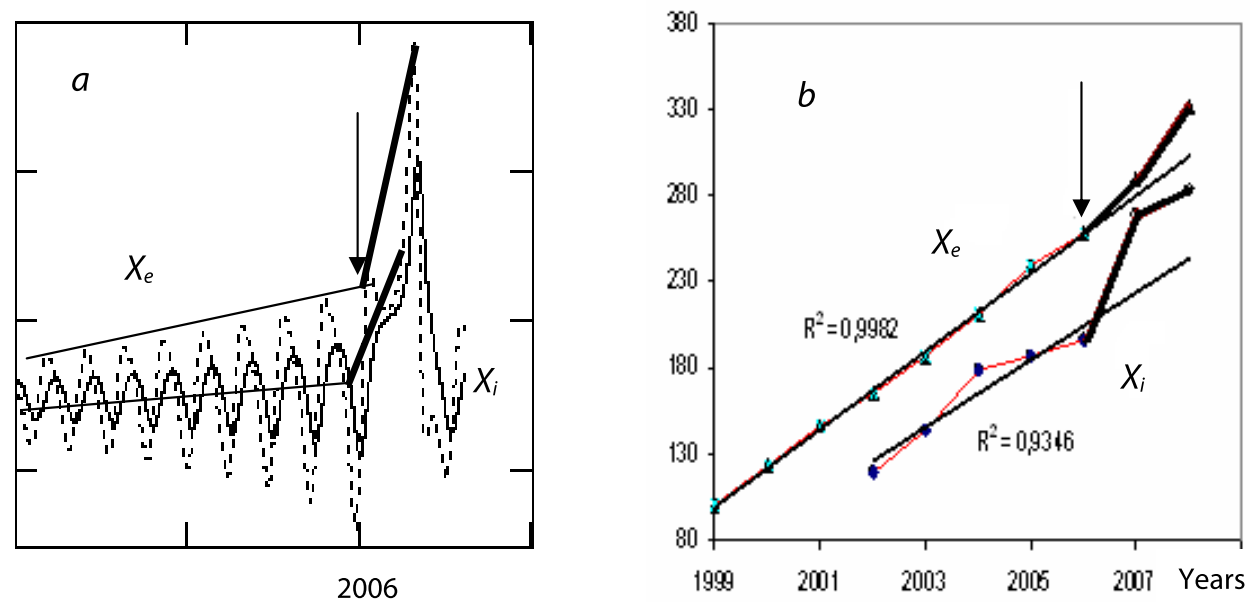

Fig. 3. Industrial producer prices in the Altai Territory $(X, 1999$ prices are taken for $100 \%)$ and consumer prices $\left(X_{e}, 1999\right.$ prices are taken for 100\%) for the products in 1999-2008; they are reduced to the same base (2000) at the first time stage: a - qualitative pattern, $b$ - statistical data

Under regression to the crisis-free condition, as follows from the figure, specific recurrence time for the prior price level is less approximately two times as much.

Fig. 4 provides a pictorial presentation related to supply and demand curves according to A. Marshall (for the specified elasticity coefficients) within the whole considered time slice for all three time stages. If the demand curve specified by a chaotic nature can be approximated as a line with sinking demand under price growth, then the supply curve for the discussed pattern corresponds to high density of possible solution paths; the given curve crosses the demand curve both above and below the point of equilibrium.

Since 2006 (marked by arrows) growth rates have undergone drastic change (increase). The pre-crisis period is described in the pattern as a smooth variation of both prices, despite they repeat periodically within the annual cycle (a broken curve for $X_{\mathrm{i}}$ in fig. $3 \mathrm{a}$ and a continuous line for $X_{\mathrm{e}}$ ).

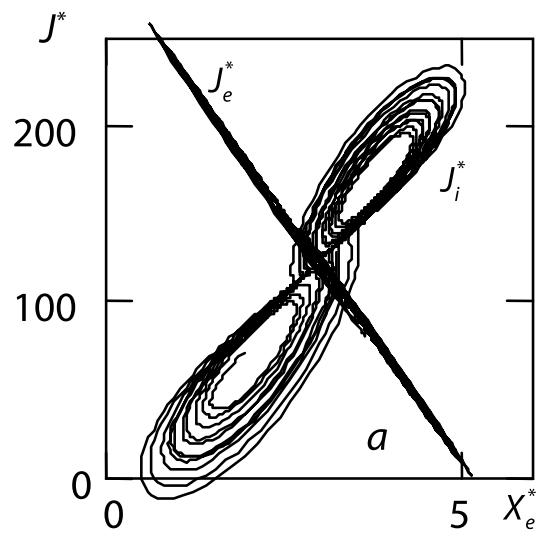

Fig. 4. Supply and demand dependences according to $A$. Marshall, restored out of the calculation in terms of Lorentz equations (with $A \sim 1$ )
Other market conditions. By changing coefficients $L_{e e}^{0^{*}}, L_{i i}^{0^{*}}, L_{i e}^{0^{*}}, L_{e i}^{0^{*}}$ we can obtain different conditions of the market (fig. 4, 5), which is specified by highelasticity or low-elasticity demand (fig. 4, fig. 5a) or another character. We can state that representation of the supply and demand curves as lines (fig. 1) is a conventional technique for market description, as there are a lot of producers on the market and each of them acts with its proper irrationality regarding both prices and volume. It is particularly visualized within a crisis period.

The given approach does not contradict the theory by A. Marshall. Price, which exceeds an equilibrium price, results in excess of supply over demand, and, as a rule, it shows up in price reduction.

Forecasting time. The given approach makes it possible to estimate reliable forecasting time regarding market state. Analysis of chaotic and regular processes within nonlinear systems generally begins with building of path difference and determination of Lyapunov exponents $\lambda$ [1]. Lyapunov exponents $\lambda$ provide information referred to divergence of two adjacent paths with barely different entry conditions. Regarding the given task, let us settle minor initial shift between supply prices $m_{0}=(\delta \mathrm{x})_{0}$. In terms of nonlinear dynamics it is called a finite order of roughening of phase space $m_{0}$. Value $(\delta x)_{0}$ is an initial distance between the two supply price paths $x^{\prime}(t)$ and $x^{\prime \prime}(t)$, which are negligibly different from each other in terms of entry conditions. With time $t$ the distance between the paths $\delta x(t)=\left|x^{\prime}(t)-x^{\prime \prime}(t)\right|$ is described by the exponent: $\delta x(t)=(\delta x)_{0} \exp (\lambda t)$, where $\lambda$ is a certain index, which is called Lyapunov exponent in a nonlinear dynamics. Reliable forecasting time regarding market state $t_{r}$ was calculated according to formula [1]: 

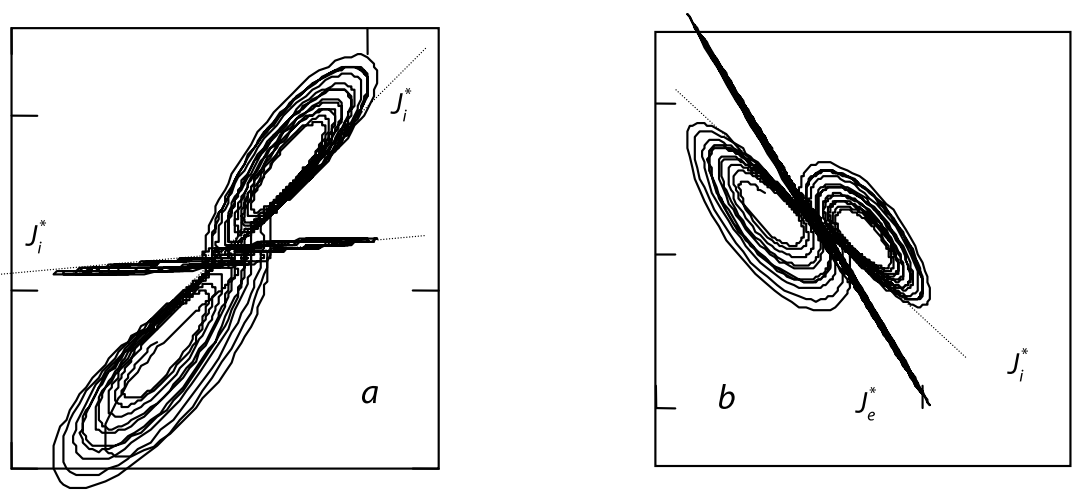

Fig. 5. A. Marshall cross for chaotic markets with different elasticity at I-III stages of crisis development (with gz/c $>>1, A \sim 1)$ : a) low-elasticity demand market nросом $\left.L_{e e}^{0^{*}}=-1, L_{e i}^{L^{*}}=5, L_{i e}^{0^{*}}=30, L_{i i}^{0^{*}} \equiv L_{i i}^{*}(0)=20 ; b\right)$ market, where both supply and demand reduce with price growth $L_{e e}^{0^{*}}=-60, L_{e i}^{L^{*}}=5, L_{i e}^{0^{*}}=-30, L_{i i}^{L^{*}} \equiv L_{i i}^{*}(0)=20$.

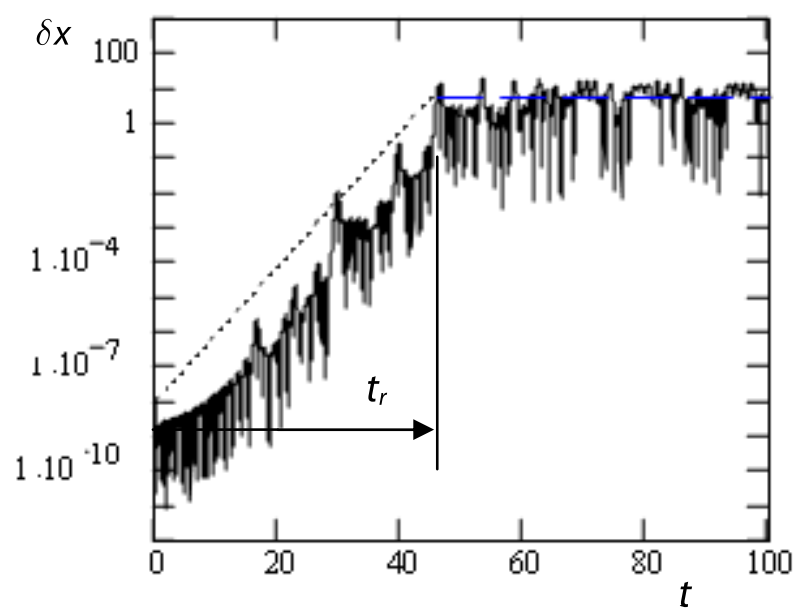

Fig. 6. Time dependence for the distance between the two

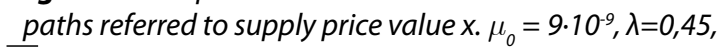
$\overline{\delta x}=5,3, t_{r}=44,9, t \equiv t / t_{0}$, time scale in fig. 3 corresponds to $t_{0}=0,35$ of the year

$$
t_{r}=\frac{1}{K_{0}} \ln \left(\frac{\overline{\delta x}}{\mu_{0}}\right),
$$

where $\overline{\delta x}-$ mean value, which was generated during the space of time tr by way of the initial value augmentation $(\delta \mathrm{x})_{0}$, - Kolmogorov entropy. For a numerical solution of a differential equation Kolmogorov entropy is equal to positive values of Lyapunov exponent $K_{0}=1$. To pass to denominate quantities, the left and right parts of expression (8) need to be multiplied by time scale $t_{0}=0,35$ of the year.

Numerical calculations have demonstrated that forecasting time for the Altai Territory is $\operatorname{tr}=15,7$ of a year, which, according to fig. 3, corresponds to 2013.

\section{References}

$\begin{array}{lllll}\text { 1. Belotserkovsky } & \text { O. M., Bystrai } & \text { G. P., Tsybulsky } & \text { V. R. }\end{array}$ Economic synergy. The issues of sustainability. Novosibirsk: Science, 2006. 116 p.

2. Indicators specifying economic and social processes in the Altai Territory: Statistical newsletter / Local authority under the Federal State Statistics Service for the Altai Territory. Barnaul, 2009. 110 p.

3. Kuznetsov S. P. Dynamic chaos. M.: Physicomathematical literature publishers, 2001. $296 \mathrm{p}$.

4. Lorents E. Determinate noncyclic motion // Strange attractors. M., 1981. p. 88-116.

5. Marshall A. Economic thought anthology. M. : ExmoPress, 2007. p. 832.

6. Chernavsky D. S., Starkov N. I., Sherbakov A. V. To the issues of physical economics. УФН. Т. 172, №9. p. 1045-1065.

7. Shuster G. Determinate chaos. Introduction. M. : Mir. 1988. 240 p.

8. Bystrai G. P. Analytical Macroeconomics: Dynamics of Non-equilibrium Economic Processes // Book of Abstracts of Interdisciplinary Integration Forum on Studies of General Systems and Applications in Engineering, Economics, Social and Other Sciences. July 13-15, 1995. p. 39-40. Slippery Rock. 